\title{
Analysis of the Cell Association for Decoupled Wireless Access in a Two Tier Network
}

\author{
Zeeshan Sattar, Joao V. C. Evangelista, Georges Kaddoum, and Naïm Batani \\ Department of Electrical Engineering \\ Ecole de technologie superieure, Montreal, Canada \\ Email: \{zeeshan.sattar.1, joao-victor.de-carvalho-evangelista.1\}@ens.etsmtl.ca \\ \{georges.kaddoum, naim.batani\}@etsmtl.ca
}

\begin{abstract}
In this paper, we analyze the association of a user terminal in a two-tier network (i.e., macrocells and millimeter wave small cells). We assumed a decoupled wireless access where a user terminal has the liberty to choose different base stations (BSs) in uplink and downlink based on the received power and the channel quality. A practical blockage model where a human body is a blockage to millimeter wave $(\mathrm{mmW})$ is considered. An in-depth simulation study is done to explore the effectiveness of decoupled wireless access in a crowded environment. In addition to that, a detailed analysis on the intuitiveness and the mathematical tractability of the blockage model used is also provided. In the end, few research questions on the efficacy of decoupled wireless access are raised in this paper.

Index Terms-Millimeter wave, fifth-generation networks, heterogeneous network, cell association, urban environment, humanbody blockage.
\end{abstract}

\section{INTRODUCTION AND MOTIVATION}

The network densification and the use of extremely high frequencies (EHF) which commonly known as millimeter wave $(\mathrm{mmW})$ band are the two most promising candidates for the future wireless access to fulfill the ever increasing demand of capacity. It is the small wavelength of $\mathrm{mmW}$ which made it practical to increase the density of BSs significantly without any increase in the absurdly large footprint of conventional BSs [1]. Though, intuitively the network densification in a heterogeneous network sounds a straight forward way to increase the capacity of an overall system but it also forces us to revisit some of the conventional techniques in cell planning and deployment of a communication system [2], [3].

Recently an idea to decouple the downlink (DL) and uplink (UL) BSs has been proposed [4], [5]. This idea not only flips the convention of coupled BSs (since the inception of mobile technology) but indirectly it also questions the way we do channel estimation as it breaks the channel reciprocity by its very design. In [4], Boccardi et al. argues on the efficacy of decoupled wireless access in hyper-dense heterogeneous networks. Though they also pointed out that without channel reciprocity in decoupled wireless access, the problem of channel estimation would become a bit more challenging, especially in case of $\mathrm{mmW}$.

It is the susceptibility to blockage of $\mathrm{mmW}$, which makes it significantly different from all other standard wireless technologies. Since, electromagnetic waves can not travel around any obstacle which exceeds their wavelength, therefore various objects which had never been considered as a blockage for microwave cause significant propagation losses for $\mathrm{mmW}$ [6]. Therefore to analyze the heterogeneous network with $\mathrm{mmW}$ BSs, it is necessary to assume a blockage model which emulates the practical scenario $\mathrm{mmW}$ faces. In the past couple of years, there has been some progress in blockage modeling for $\mathrm{mmW}$ wireless access [7], [8], [9].In this paper, we are using a very recently proposed blockage model which quantifies the effect of th human body on $\mathrm{mmW}$ [10] to analyze the cell association in a decoupled wireless access.

The proposal of decoupled wireless access is getting considerable attention since its inception [11] and authors in [4], [5] made quite reasonable arguments in its favor. In this paper, we explore the efficacy of decoupled wireless access in an environment where the human body is considered as a blockage to $\mathrm{mmW}$ wireless link. Since, highly populated areas would be the one which will attract the deployment of $\mathrm{mmW}$ network to fulfill the ever increasing demand of wireless traffic, therefore, it is very important to study the effects of the human body on the decoupled wireless access.

The rest of the paper is organized as follows. In Section II, we describe the system model in detail, which includes the propagation assumptions and a precise description of the blockage model used. In Section III, a commentary on the mathematical feasibility of the considered blockage model is provided. In Section IV, discussion on the obtained simulation results is provided and Section V concludes the paper.

\section{SySTEM MOdEL}

The system model consists of a two-tier heterogeneous cellular network, where sub-6GHz (i.e., conventional microwave or mcell) BSs and $\mathrm{mmW}$ (i.e., scell) BSs are modeled using independent homogeneous Poisson point process (PPP) as shown in Fig.1 . All the BSs are uniformly distributed in an area of concern (a circular area with radius $\mu$ ). We use $\Phi_{k}$ to denote the set of points obtained through PPP with density $\lambda_{k}$, that can be explicitly written as

$$
\Phi_{k} \triangleq\left\{x_{k, i} \in \mathbb{R}^{2}: i \in \mathbb{N}_{+}\right\}, \quad k \in \mathcal{K},
$$

where set $\mathcal{K} \triangleq\{$ scell,mcell $\}$. In addition to that all the user equipments (UEs) are assumed to form an independent PPP with density $\lambda_{u}$ and they are denoted by a set $\Phi_{u}$ given as 


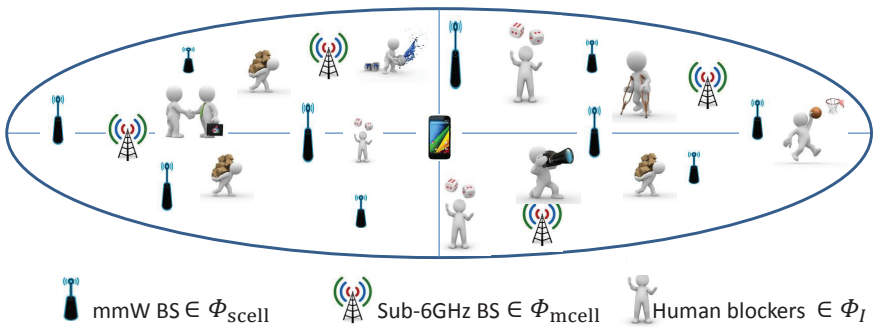

Fig. 1: System Model

$$
\Phi_{u} \triangleq\left\{u_{j} \in \mathbb{R}^{2}: j \in \mathbb{N}_{+}\right\} .
$$

Since the distribution of a point process is completely indifferent to the addition of a node at the origin, thanks to Slivnyak's theorem [12], the analysis is done for a typical UE located at origin $u_{j}=(0,0)$.

The summary of parameters and notations used in the rest of this paper is presented in Table I

TABLE I: System parameters and their definitions

\begin{tabular}{|c|c|}
\hline Notation & Description \\
\hline$P_{u k}$ & $\begin{array}{l}\text { UE transmit power to } \mathrm{BS} \text { in } k^{t h} \text { tier, where } k \in \\
\{\text { scell, mcell }\}\end{array}$ \\
\hline$P_{k}$ & $\begin{array}{l}\text { Transmit power of } \mathrm{BS} \text { in } k^{\text {th }} \text { tier, where } k \in \\
\{\text { scell, mcell }\}\end{array}$ \\
\hline $\begin{array}{c}T_{k}, T_{k}^{\prime} \\
\psi_{k}\end{array}$ & $\begin{array}{l}\text { DL and UL association bias for } k \in\{\text { scell, mcell }\} \\
\text { The combination of antenna gain and near-field pathloss } \\
\text { for } k \in\{\text { scell, mcell }\}\end{array}$ \\
\hline$L_{\min , k}$ & $\begin{array}{l}\text { The minimum pathloss }\|x\|^{\alpha_{k}} \text { of the typical UE from } \\
\text { the } k^{t h} \text { tier }\end{array}$ \\
\hline$\alpha_{k}$ & $\begin{array}{l}\text { The pathloss exponent, for macrocell i.e., when } k= \\
m \text { cell its value remains constant. On the other hand for } \\
k=\text { scell, pathloss exponent becomes a function of the } \\
\text { distance between the transmitter and the receiver, and its } \\
\text { value switches between line of sight (LOS) and non line } \\
\text { of sight (NLOS) exponent values with the probability } \\
P_{\text {LOS }} \text { and } P_{\text {NLOS }} \text {, respectively }\end{array}$ \\
\hline
\end{tabular}

\section{A. Propagation assumptions and cell association criteria}

In our system model it is assumed that all the UEs and sub$6 \mathrm{GHz}$ BSs have omni directional antennas and antenna gains from a massive array of antenna elements are only accounted for the mmW BSs. It is a realistic assumption in a sense that in such hybrid BSs' deployment, the sub-6GHz BSs will provide an umbrella coverage to all the UEs to guarantee a consistent service, whereas the $\mathrm{mmW}$ BSs will mainly focus on high capacity link with individual UEs. Therefore the antenna gain is only considered with $\mathrm{mmW}$ BS.

It is assumed that in both UL and DL, a typical UE associates with a BS based on the received power. The typical UE associates with a BS in UL at $x * \in \Phi_{l}$, where $l \in\{$ scell, mcell $\}$ if and only if

$$
\begin{aligned}
P_{u l} T_{l}^{\prime} \psi_{l} L_{l}(x *)^{-1} \geq \quad & P_{u k} T_{k}^{\prime} \psi_{k} L_{\min , k}^{-1}, \\
& \forall k \in\{\text { scell, mcell }\} .
\end{aligned}
$$

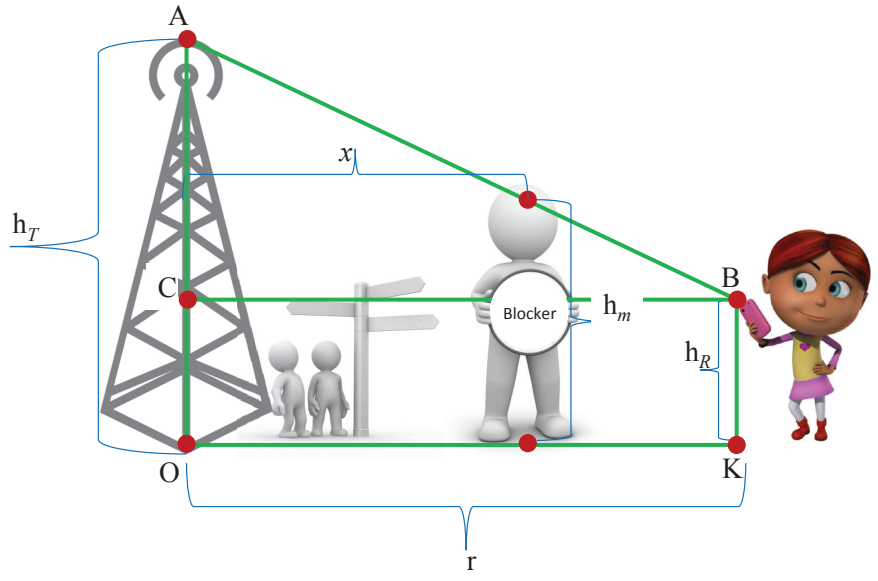

Fig. 2: Blockage Scenario

Similarly, a typical UE associates with a BS in DL at $x * \epsilon$ $\Phi_{l}$ if and only if

$$
\begin{aligned}
P_{l} T_{l}^{\prime} \psi_{l} L_{l}(x *)^{-1} \geq & P_{k} T_{k}^{\prime} \psi_{k} L_{\min , k}^{-1}, \\
& \forall k \in\{\text { scell, mcell }\} .
\end{aligned}
$$

\section{B. Blockage Model}

In this paper, we use a very intuitive blockage model [10], where a human body is considered as blockage to $\mathrm{mmW}$. The potential blockers are generated using a independent homogeneous PPP $\Phi_{I}$ over the area of concern with intensity $\lambda_{I}$ as shown in Fig. 1. Each blocker is modeled as a cylinder with a certain height $H$ and a width $W$. Here, both the height and the width are generated randomly using the well researched statistical data [13].

Moreover, it is obvious from Fig. 2 that not all blockers can affect the LOS link between transmitter and receiver. Therefore, we can model the PPP of blockers whose height can cause the LOS link to break by thinning the $\Phi_{I}$ with probability $\operatorname{Pr}\left(H>h_{m}(x)\right)$. The thinned PPP is denoted as $\Phi_{I B}$ with density $\lambda_{I B}$,

$$
\lambda_{I B}(x)=\lambda_{I} \operatorname{Pr}\left(H>h_{m}(x)\right), \quad x \in(0, r),
$$

where $h_{m}(x)$ is a function describing the distance between the LOS link and the ground at $x$

$$
h_{m}(x)=-\frac{h_{T}-h_{R}}{r} x+h_{T} .
$$

And as shown in Fig. 2, $h_{T}$ and $h_{R}$ are the Tx and Rx heights, respectively. The aforementioned process $\Phi_{I B}$ is nonhomogeneous but still remains Poisson with thinned density $\lambda_{I B}(x)$, which increases non-linearly as $x$ grows [12]. The probability $\operatorname{Pr}\left(H>h_{m}(x)\right)$ is a complementary cumulative distribution function (CCDF) of $H$. Since, $H$ follows Normal distribution [13], The probability $\operatorname{Pr}\left(H>h_{m}(x)\right)$ takes the following form

$$
\operatorname{Pr}\left(H>h_{m}(x)\right)=1-\frac{1}{2}\left[1+\operatorname{erf}\left(\frac{h_{m}(x)-\mu_{H}}{\sigma_{H} \sqrt{2}}\right)\right],
$$




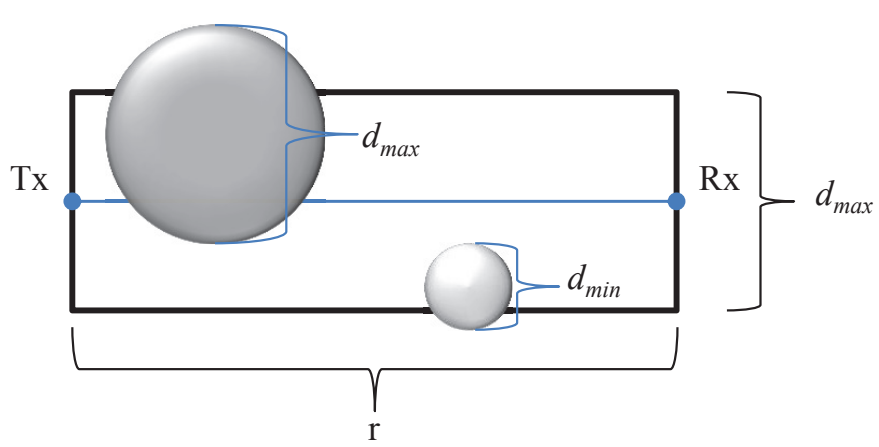

Fig. 3: Top view of the blockage scenario

where $\operatorname{erf}($.$) is the error function, \mu_{H}, \sigma_{H}$ are mean and variance of $H$, respectively.

For the mathematical formulation of probability of LOS $\left(P_{\mathrm{LOS}}\right)$ we have to determine the probability of few events described in Table II. Having defined all the events and

TABLE II: Probabilistic events and their definitions

\begin{tabular}{c|l}
\hline Events & Description \\
\hline \hline$A_{i}$ & There are $i$ blockers in the area of interest \\
$B_{0}$ & Diameter of the blocker is not large enough to cross the \\
& LOS link \\
$B_{1}$ & Complementary to $B_{0}$ \\
$C_{0}$ & Blocker's height is not large enough to block the LOS \\
& link \\
$C 1$ & Complementary to $C_{0}$ \\
\hline
\end{tabular}

probabilities the expression for $P_{\mathrm{LOS}}$ can be formulated as follows:

$$
\begin{aligned}
P_{\mathrm{LOS}}= & \operatorname{Pr}\left\{A_{0}\right\}+\sum_{i=1}^{\infty} \operatorname{Pr}\left\{A_{i}\right\} \\
& \cdot\left[\operatorname{Pr}\left\{B_{0}\right\}+\operatorname{Pr}\left\{B_{1}\right\} \operatorname{Pr}\left\{C_{0}\right\}\right]^{i},
\end{aligned}
$$

where the first part of the equation $\operatorname{Pr}\left\{A_{0}\right\}$ is the probability that there are no blockers in the area of interest and the second part of the equation sums the probability in the event that there are $i$ blockers in the area of interest, but their width and height are not enough to block the LOS link. A rectangular area shown in Fig. 3 is considered where the widths of all blockers are uniformly distributed between $d_{\min }$ and $d_{\max }$, therefore, the width of this area is bounded by $d_{\max }$. As mentioned in the start of this section, the number of blockers follow a Poisson distribution, hence, the number of blockers in the area of concern follows a Poisson distribution with the intensity $\lambda_{I} r d_{\max }$. Having defined all the necessary assumptions, the mathematical expressions of aforementioned events can be easily formulated as in [10].

Even though this blockage model is intuitive in nature and it can accurately emulates a crowded environment [10], so far, there is no closed-form expression for this model. To the best of our knowledge, it is mathematically intractable to provide a closed-form expression of $P_{\mathrm{LOS}}$, as its expression contains double integral of erf function. Since, it is a well known fact that, mathematically, it is extremely difficult to approximate an integral of erf function over a wide range of values (which is the case here). Hence, further discussion on the efficacy, intuitiveness, and mathematical intractability of this blockage model in calculating the association probabilities is provided in the following section.

\section{Analytical Analysis}

In this section expressions for the association probability for a typical user in the human blockage model scenario are derived. On the association scenario under analysis there are two random variables to be considered; they are the associated tier for uplink $\mathcal{A}_{\mathrm{UL}}$ and the associated tier for downlink $\mathcal{A}_{\mathrm{DL}}$. Considering the model proposed in Section II with two tiers there are four possible outcomes:

- $\mathcal{A}_{\mathrm{UL}}=$ mcell, $\mathcal{A}_{\mathrm{DL}}=$ mcell

- $\mathcal{A}_{\mathrm{UL}}=$ mcell, $\mathcal{A}_{\mathrm{DL}}=$ scell

- $\mathcal{A}_{\mathrm{UL}}=$ scell, $\mathcal{A}_{\mathrm{DL}}=$ mcell

- $\mathcal{A}_{\mathrm{UL}}=$ scell, $\mathcal{A}_{\mathrm{DL}}=$ scell

As the events $\mathcal{A}_{\mathrm{UL}}$ and $\mathcal{A}_{\mathrm{DL}}$ are independent, the derivation of the probabilities $\operatorname{Pr}\left(\mathcal{A}_{\mathrm{UL}}=m c e l l\right), \operatorname{Pr}\left(\mathcal{A}_{\mathrm{UL}}=\right.$ scell $)$, $\operatorname{Pr}\left(\mathcal{A}_{\mathrm{DL}}=m c e l l\right)$ and $\operatorname{Pr}\left(\mathcal{A}_{\mathrm{DL}}=\right.$ scell $)$ are enough to calculate the probabilities of the four possible outcomes. Furthermore, the user only associates to one base station for uplink and one for downlink, therefore

$$
\operatorname{Pr}\left(\mathcal{A}_{\mathrm{UL}}=\text { mcell }\right)=1-\operatorname{Pr}\left(\mathcal{A}_{\mathrm{UL}}=\text { scell }\right),
$$

and

$$
\operatorname{Pr}\left(\mathcal{A}_{\mathrm{DL}}=\text { mcell }\right)=1-\operatorname{Pr}\left(\mathcal{A}_{\mathrm{DL}}=\text { scell }\right) .
$$

As seen in equations (1) and (2) the base station to which the user associates depends on the minimum path loss of the typical UE from the $k^{t h}$ tier, so, in order to derive the probabilities of association, the point process obtained from the path loss between the typical user and each base station must be characterized. Following a similar approach to [5], the path loss point process is defined as

$\mathcal{P}_{k}:\left\{L_{k}(x)=\|x\|^{\alpha_{k}(\|x\|)}\right\}_{x \in \Phi_{k}}$, for $k \in\{$ scell, mcell $\}$.

From the displacement theorem [14], $\mathcal{P}_{k}$ is a Poisson point process with intensity measure $\Lambda_{k}(\cdot)$ and CCDF

$$
\bar{F}_{L_{k}}(t)=\operatorname{Pr}\left(L_{k}(x) \geq t\right)=\exp \left(-\Lambda_{k}([0, t]) .\right.
$$

Lemma 1. The intensity measure of the path loss process of the tagged BS for tiers 1 and 2 are given by

$$
\begin{aligned}
\Lambda_{m}([0, t])= & \pi \lambda_{m} t^{\frac{2}{\alpha_{m}}} \\
\Lambda_{s}([0, t])= & 2 \pi \lambda_{s}\left[\int_{0}^{t^{\frac{1}{\alpha_{L O S}}}} r P_{L O S}(r) d r\right. \\
& \left.+\int_{0}^{t^{\frac{1}{\alpha_{L L O S}}}} r\left(1-P_{\operatorname{LOS}}(r)\right) d r\right] .
\end{aligned}
$$


Proof. The proof for the macro cell case (10) is available on [5]. For the scell (mmWave) we have that the intensity of the path loss process $\mathcal{P}_{s}:\left\{L_{s}(x)=\|x\|^{\alpha_{s}(\|x\|)}\right\}_{x \in \Phi_{s}}$ is given by

$$
\Lambda_{s}([0, t])=\lambda_{s} \int_{\mathbb{R}^{2}} \operatorname{Pr}\left(L_{s}(x)<t\right) d x .
$$

Switching to polar coordinates leads to

$$
\Lambda_{s}([0, t])=2 \pi \lambda_{s} \int_{0}^{\infty} \operatorname{Pr}\left(r^{\alpha_{2}(r)}<t\right) r d r .
$$

As described in Section II $\alpha_{s}(r)$ is equal to $\alpha_{\mathrm{LOS}}$ with probability $P_{\mathrm{LOS}}$ and $\alpha_{\mathrm{NLOS}}$ with probability $1-P_{\mathrm{LOS}}$. Thus, we have

$$
\begin{aligned}
\Lambda_{s}([0, t]) & =2 \pi \lambda_{s}\left[\int_{0}^{\infty} r P_{\mathrm{LOS}}(r) \mathbb{1}\left(r<t^{\frac{1}{\alpha_{\mathrm{LOS}}}}\right) d r\right. \\
& \left.+\int_{0}^{\infty} r\left(1-P_{\mathrm{LOS}}(r)\right) \mathbb{1}\left(r<t^{\frac{1}{\alpha_{\mathrm{NLOS}}}}\right) d r\right]
\end{aligned}
$$

which leads to (11).

From (10) and (9) it is possible to obtain the probability density function (PDF) as

$$
f_{L_{m}}(t)=-\frac{d \bar{F}_{m}(t)}{d t}=\frac{2 \pi \lambda_{m} t^{\frac{2}{\alpha_{m}}}-1}{\alpha_{m}} \exp \left(-\pi \lambda_{m} t^{\frac{2}{\alpha_{m}}}\right) .
$$

For the millimeter wave scell tier the CCDF is given by

$$
\begin{aligned}
\bar{F}_{L_{s}}(t)=\exp [ & -2 \pi \lambda_{s}\left(\int_{0}^{t^{\frac{1}{\alpha_{\mathrm{LOS}}}}} r P_{\mathrm{LOS}}(r) d r\right. \\
& +\int_{0}^{\left.\left.t^{\frac{1}{\alpha_{\mathrm{NLOS}}}} r\left(1-P_{\mathrm{LOS}}(r)\right) d r\right)\right] .} .
\end{aligned}
$$

By manipulating (1) and (2) it is possible to obtain an expression for the probability of associating to the macro cell in the uplink and in the downlink as

$$
\begin{aligned}
\operatorname{Pr}\left(\mathcal{A}_{\mathrm{UL}}=\text { mcell }\right) & =\operatorname{Pr}\left(L_{\mathrm{min}, \text { scell }}>a_{\mathrm{UL}} L_{\text {min }, \text { mcell }}\right) \\
& =\frac{1}{a_{\mathrm{UL}}} \int_{0}^{\infty} \bar{F}_{L_{s}}(l) f_{L_{m}}\left(\frac{l}{a_{\mathrm{UL}}}\right) d l
\end{aligned}
$$

and

$$
\begin{aligned}
\operatorname{Pr}\left(\mathcal{A}_{\mathrm{DL}}=\text { mcell }\right) & =\operatorname{Pr}\left(L_{\mathrm{min}, \text { scell }}>a_{\mathrm{DL}} L_{\mathrm{min}, \text { mcell }}\right) \\
& =\frac{1}{a_{\mathrm{DL}}} \int_{0}^{\infty} \bar{F}_{L_{s}}(l) f_{L_{m}}\left(\frac{l}{a_{\mathrm{DL}}}\right) d l
\end{aligned}
$$

where

$$
a_{\mathrm{UL}}=\frac{P_{u, \text { scell }} T_{\text {scell }}^{\prime} \psi_{\text {scell }}}{P_{u, \text { mcell }} T_{\text {mcell }}^{\prime} \psi_{m c e l l}},
$$

and

$$
a_{\mathrm{DL}}=\frac{P_{\text {scell }} T_{\text {scell }} \psi_{\text {scell }}}{P_{\text {mcell }} T_{\text {mcell }} \psi_{\text {mcell }}} .
$$

As the calculation of $P_{\mathrm{LOS}}(r)$ is not obtained in closedform and involves the numerical evaluation of three integrals, it is not feasible to obtain a closed-form expression for $\bar{F}_{L_{s}}(t)$ as well. Considering the intractability of calculating $\bar{F}_{L_{s}}(l)$ due to the blockage model, a simulation approach is taken to characterize the probabilities of association under this blockage model.

\section{Simulation Results}

\section{A. Simulation setup}

A system-level simulation model is developed to mimic the real scenario of association between a UE and its tagged BS(s) in a decoupled wireless access environment. The simulation model not only provides the association probabilities of a particular UE with its tagged BS(s) but it also gives an insight on the portability of having a decoupled wireless access.

We generated the blockers, the $\mathrm{mmW}$ BSs, and the sub$6 \mathrm{GHz}$ BSs in a circular area of radius $\mu$ as described in Section II. In case of $\mathrm{mmW}$ wireless access, for the sake of consistency with previous published work [10] the height of the transmitter and the receiver are assumed to be $4 \mathrm{~m}$ and $1.3 \mathrm{~m}$, respectively. And as described in section II-B, each generated blocker has a random height and width, following [10], the height and width of the blockers are generated using normal $\mathcal{N}\left(\mu_{H}, \sigma_{H}\right)$ and uniform $\mathcal{U}\left(d_{\min }, d_{\max }\right)$ distributions, respectively. Here, $\mu_{H}, \sigma_{H}, d_{\min }$, and $d_{\max }$ are assumed to be $1.7 \mathrm{~m}, 0.1 \mathrm{~m}, 0.2 \mathrm{~m}$, and $0.8 \mathrm{~m}$, respectively. The rest of the parameters used in the simulation are same as listed in [5].

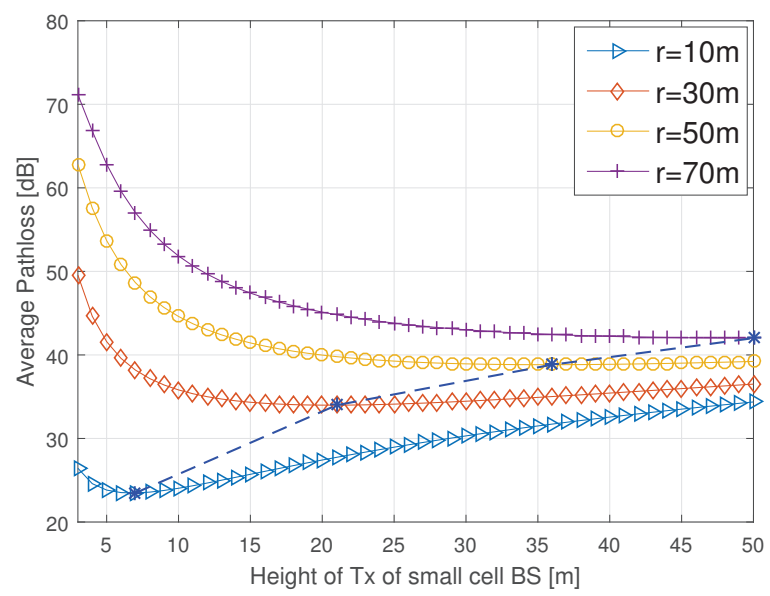

Fig. 4: Average pathloss vs optimal Tx height for different $r$

\section{B. Discussion}

As already mentioned in III that the blockage model under consideration has no closed-form expression and this fact made it mathematically intractable for further analytical analysis. Nevertheless, its practical nature is still very useful. For 


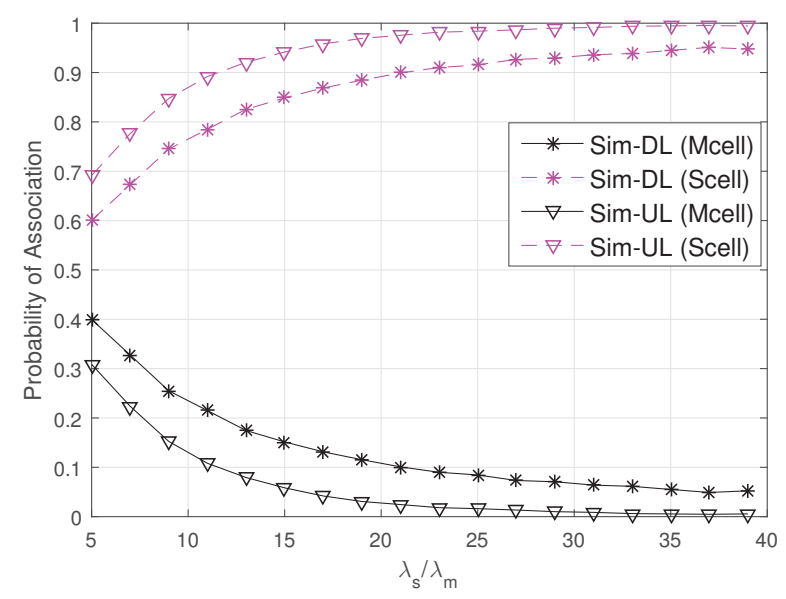

Fig. 5: Association probability for antenna gain $=30 \mathrm{dBi}$, and blockers intensity $\lambda_{I}=0.3$ blockers $/ \mathrm{m}^{2}$

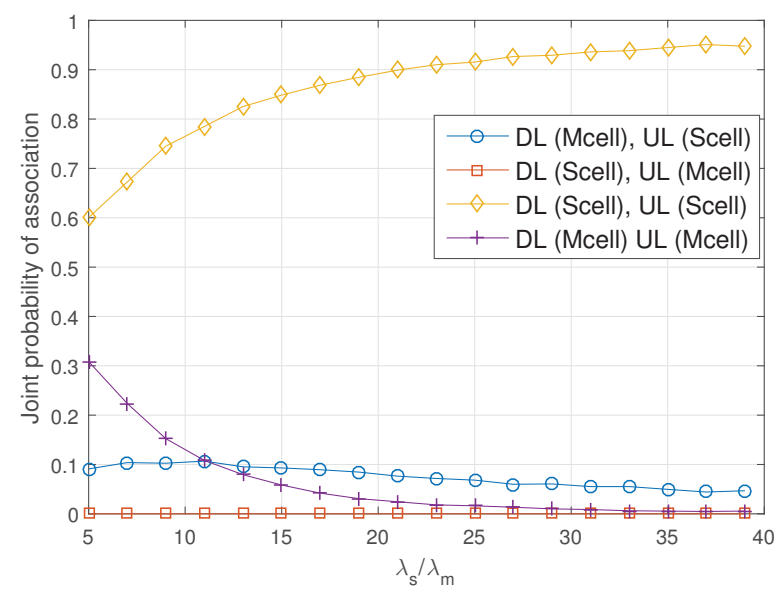

Fig. 6: Joint association probabilities for antenna gain $=30 \mathrm{dBi}$, and blockers intensity $\lambda_{I}=0.3$ blockers $/ m^{2}$

example, it is obvious that the average distance between the transmitter and the receiver is a function of the intensity of blockers $\lambda_{I}$. It implies that the optimal height of the Tx of scell BS to minimize the average pathloss is also a function of $\lambda_{I}$, as the optimal height of the Tx depends on the average distance between Tx and Rx. Using this blockage model we can easily predict the optimal height of the Tx of scell BSs for different urban environments as shown in Fig. 4. The dashed curve in Fig. 4, which is intersecting all the other curves shows the optimal height of the Tx for different values of distance $r$. Here, we want to emphasize on the fact that to choose the optimal height of the Tx in scell is extremely important, because it makes a huge difference in average pathloss. Therefore, any arbitrary height of the Tx can make or break the connection completely. Hence, in our opinion Txs for next generation of wireless access should be designed to adjust their heights in real-time according to the density of blockers $\lambda_{I}$.

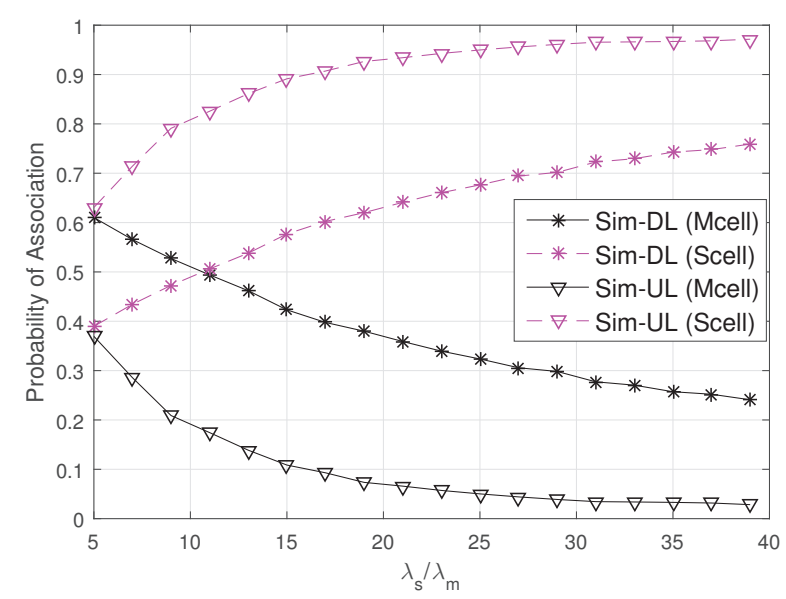

Fig. 7: Association probability for antenna gain $=18 \mathrm{dBi}$, and blockers intensity $\lambda_{I}=0.3$ blockers $/ \mathrm{m}^{2}$

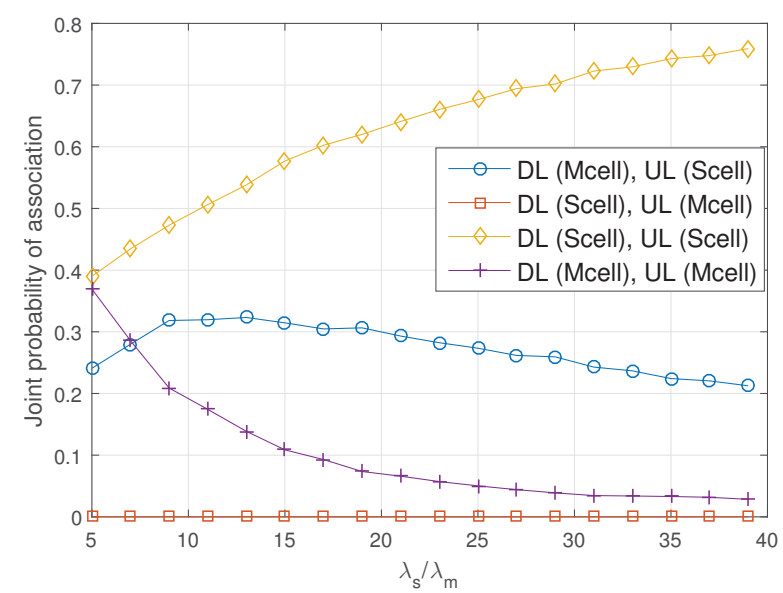

Fig. 8: Joint association probabilities for antenna gain $=18 \mathrm{dBi}$, and blockers intensity $\lambda_{I}=0.3$ blockers $/ \mathrm{m}^{2}$

The association probabilities of a UE with two tiers of BSs are shown in Fig. 5 and Fig. 7. Whereas Fig. 6 and Fig. 8 show the joint probabilities of four possible association scenarios of a particular UE as mentioned in Section III. It is obvious from the simulation results in Fig. 5, Fig. 6, Fig. 7, and Fig. 8 that antenna gain plays a significant role on the efficacy of decoupled wireless access. A higher antenna gain (which would be the case in future $\mathrm{mmW}$ BSs) significantly reduces the decoupling gain (i.e., when a UE chooses to select two different types of BSs in DL and UL).

Moreover, an interesting observation which we can be made from Fig. 6 and Fig. 8 is that the joint association probability of an event when a particular UE connects to scell in DL and mcell in UL is zero. This contradicts with the argument made in [4] in support of decoupled wireless access; that is for future generation of wireless network, more UEs will connect with scell in DL for higher data rate and mcell will provide an umbrella coverage as well as UL connection to 
decoupled UEs. Though, their argument was based on the difference between the allowed transmit power for $\mathrm{mmW}$ and microwave UEs, but even that difference would not be enough to make the joint probability of aforementioned event to nonzero [15]. Mathematically, using equations (15) and (16) we can formulate the joint probability of the aforementioned event as $\operatorname{Pr}\left(L_{\min , s}<a_{\mathrm{DL}} L_{\min , m} ; L_{\min , s}>a_{\mathrm{UL}} L_{\min , m}\right)$. Since, in general $a_{\mathrm{UL}}>a_{\mathrm{DL}}$, therefore the joint association probability of that event will remain zero.

Even though, the power biasing can be used to change the inequality $a_{\mathrm{UL}}>a_{\mathrm{DL}}$ and also to do the load balancing between the two tiers of network, which would definitely increase the decoupling gain but it would also result in decrease in the sum-capacity of overall network. Besides that, as decoupled wireless access kills the channel reciprocity by its very design, which would force network to bear the additional cost of control signals. Therefore, the answer to the question of how much pragmatic this idea can be for the future wireless network will solely depends on an in depth cost analysis of additional control signals. Furthermore, if the industry decides to stick with the time division duplexing (TDD), we believe that the future of decoupled wireless access will remain ambiguous.

\section{CONCLUSION}

We derive following conclusions from this study. Firstly, despite the fact that the considered blockage model is mathematically intractable, which makes it infeasible for stochastic geometric analysis of wireless networks, still its practical nature provides some interesting insights. Such as, tuning of Tx height with respect to the density of blockers or association biasing for load balancing. Secondly, the decoupling gain in our studied scenario is not very significant. Therefore, whether we should bear the cost of extra control signaling for decoupled wireless access solely depends on a cost analysis of additional control signals, and only then anything on its pragmatism can be said. Lastly, if TDD becomes a standard for next generation of wireless network, then the future of decoupled wireless access is certainly very bleak.

\section{ACKNOWLEDGMENT}

This work was partially supported by Mitacs through the Mitacs Globalink graduate fellowship.

\section{REFERENCES}

[1] J. G. Andrews, S. Buzzi, W. Choi, S. V. Hanly, A. Lozano, A. C. Soong, and J. C. Zhang, "What will 5g be?," IEEE J. Sel. Areas Commun., vol. 32, no. 6, pp. 1065-1082, 2014.

[2] J. G. Andrews, "Seven ways that hetnets are a cellular paradigm shift," IEEE Commun. Mag., vol. 51, no. 3, pp. 136-144, 2013.

[3] F. Boccardi, R. W. Heath, A. Lozano, T. L. Marzetta, and P. Popovski, "Five disruptive technology directions for 5g," IEEE Commun. Mag., vol. 52, no. 2, pp. 74-80, 2014.

[4] F. Boccardi, J. Andrews, H. Elshaer, M. Dohler, S. Parkvall, P. Popovski, and S. Singh, "Why to decouple the uplink and downlink in cellular networks and how to do it," IEEE Commun. Mag., vol. 54, no. 3, pp. 110-117, 2016.

[5] H. Elshaer, M. N. Kulkarni, F. Boccardi, J. G. Andrews, and M. Dohler, "Downlink and uplink cell association with traditional macrocells and millimeter wave small cells," IEEE Trans. Wireless Commun., vol. 15, no. 9, pp. 6244-6258, 2016.

[6] P. Adhikari, "Understanding millimeter wave wireless communication," Loea Corporation, 2008.

[7] T. Bai, R. Vaze, and R. W. Heath, "Analysis of blockage effects on urban cellular networks," IEEE Trans. Wireless Commun., vol. 13, no. 9 , pp. 5070-5083, 2014.

[8] S. Singh, M. N. Kulkarni, A. Ghosh, and J. G. Andrews, "Tractable model for rate in self-backhauled millimeter wave cellular networks," IEEE J. Sel. Areas Commun., vol. 33, no. 10, pp. 2196-2211, 2015.

[9] M. N. Kulkarni, S. Singh, and J. G. Andrews, "Coverage and rate trends in dense urban mmwave cellular networks," in Proc. Global Communications Conference (GLOBECOM), pp. 3809-3814, IEEE, 2014.

[10] M. Gapeyenko, A. Samuylov, M. Gerasimenko, D. Moltchanov, S. Singh, E. Aryafar, S.-p. Yeh, N. Himayat, S. Andreev, and Y. Koucheryavy, "Analysis of human-body blockage in urban millimeter-wave cellular communications," in Proc. IEEE International Conference on Communications (ICC), pp. 1-7, IEEE, 2016.

[11] H. Elshaer, F. Boccardi, M. Dohler, and R. Irmer, "Downlink and uplink decoupling: A disruptive architectural design for 5g networks," in Proc. of IEEE Global Communications Conference (GLOBECOM), pp. 17981803, IEEE, 2014.

[12] S. N. Chiu, D. Stoyan, W. S. Kendall, and J. Mecke, Stochastic geometry and its applications. John Wiley \& Sons, 2013.

[13] C. L. Ogden, C. D. Fryar, M. D. Carroll, and K. M. Flegal, Mean body weight, height, and body mass index: United States 1960-2002. Department of Health and Human Services, Centers for Disease Control and Prevention, National Center for Health Statistics Washington, DC, 2004.

[14] B. Baszczyszyn, M. K. Karray, and H. P. Keeler, "Using poisson processes to model lattice cellular networks," in Proc. IEEE INFOCOM, pp. 773-781, April 2013.

[15] D. Colombi, B. Thors, and C. Törnevik, "Implications of emf exposure limits on output power levels for $5 \mathrm{~g}$ devices above $6 \mathrm{ghz}$," IEEE Antennas Wireless Propag. Lett., vol. 14, pp. 1247-1249, 2015. 